\title{
Dolor mandibular de origen cardíaco
}

\author{
Sáez Yuguero MR*, Bermejo Fenoll A**, Calvo Guirado JL*, Álvarez Martínez E***
}

\section{RESUMEN}

En las complejas estructuras anatómicas orofaciales, asientan muchos procesos dolorosos. Las algias orofaciales más comunes tienen su origen a nivel dental, periodontal o en estructuras musculoesqueleticas. Sin embargo, el paciente puede manifestar dolor en esta región, bien en dientes o estructuras musculoesqueleticas, y la fuente originaria localizarse a distancia. A este tipo de dolor se le llama dolor heterotópico. Una posible fuente de dolor heterotópico es el dolor de origen cardíaco.

Este articulo presenta un caso clínico en el que el dolor mandibular bilateral fue el síntoma inicial de una cardiopatía isquémica que fue erróneamente etiquetada de artrosis de la articulación temporomandibular.

Palabras clave: Dolor cardiaco, dolor mandibular, dolor heterotópico.

\section{SUMMARY}

In the complex orofacial anatomic structures, many painful processes are felt. The most common orofacial pains have their origin at dental or periodontal level, or in muscle -skeletical structures. However, the patient can have pain in this area- either in teeth or muscle-eskeletical structures- and the originating source can be located at a distance. This type of pain is called heterotopic pain. A possible source of heterotopic pain is the pain of cardiac origin.

This paper presents a clinical case in which the bilateral mandibular pain is the initial symptom of an ischemic cardiopathy that was erroneously labeled as temporomandibular arthrosis.

Key words: Cardiac pain, mandibular pain, heterotopic pain.

Aceptado para publicación: Abril 2003.

* Profesora Asociada de Clínica Odontológica Integrada de Adultos. Facultad de Medicina y Odontología. Universidad de Murcia.

** $\quad$ Catedrático de Medicina Bucal. Facultad de Medicina y Odontología. Universidad de Murcia.

*** Odontólogo. Cirujano maxilofacial. Universidad de Antioquía. Hospital San Vicente de Paúl. Medetillín (Colombia).

Sáez Yuguero MR, Bermejo Fenoll A, Calvo Guirado JL, Álvarez Martínez E. Dolor mandibular de origen cardíaco. Av. Odontoestomatol 2003; 19-5: 219-223.

\section{IINTRODUCCIÓN}

dolor referido a las estructuras orofaciales en muchas ocasiones supone un importante desafío diagnóstico para el odontólogo o el médico.

Un alto porcentaje de la semiología dolorosa que se manifiesta en el macizo craneofacial, tienen su ori- gen en los problemas estomatológicos, especialmente en las estructuras dentarias, sin embargo, es de vital importancia saber que en las estructuras faciales, pueden localizarse dolores heterotópicos o dolores referidos de un problema orgánico distante.

Algunos problemas cardiovasculares y especialmente la isquemia aguda del miocardio, puede tener 
representación dolorosa en la región orofacial y ser el síntoma único inicial o bien ser la queja principal del paciente.

El profesional, odontólogo o médico, debe conocer e identificar el dolor de origen cardiaco referido a áreas orofaciales, especialmente en personas con factores de riesgo, y realizar un exhaustivo diagnostico diferencial, lo que puede ser vital para el paciente si no se actúa a tiempo.

Debemos establecer un diagnóstico clínico apropiado, para que el tratamiento se dirija hacia la fuente del dolor y no al sitio donde se manifiesta el dolor.

Este artículo revisa la literatura relacionada con el dolor referido de origen cardíaco y presenta un caso clínico de dolor mandibular como síntoma inicial único, originado por un angor inestable a consecuencia de la lesión severa de dos vasos coronarios.

\section{FISIOPATOLOGÍA DEL DOLOR CARDIACO}

La isquemia es una situación producida por la deprivación de oxígeno y la eliminación inadecuada de los metabolitos; desde un punto de vista práctico, la isquemia del miocardio se debe casi siempre a una disminución del flujo sanguíneo a través de las arterias coronarias.

Por este motivo, las manifestaciones clínicas y las consecuencias anatomopatológicas de la isquemia coronaria se denominan indistintamente cardiopatía isquémica o enfermedad coronaria.

El mecanismo que provoca la isquemia no siempre es el mismo; con frecuencia se trata de un aumento de las necesidades de oxígeno, provocado por los cambios en la presión arterial y la frecuencia cardiaca (ejercicio y emociones), en un paciente con lesiones aterosclerosas coronarias, mientras que en otras ocasiones el dolor sobreviene sin causa aparente, sugiriendo que se ha producido una reducción espontánea del aporte de oxígeno.

La isquemia provoca la liberación celular de sustancias como la serotonina, la histamina o la bradicinina y permite la acumulación de metabolitos ácidos y de potasio; se cree que alguna de estas sustancias estimula las terminaciones nerviosas y provoca el dolor característico de la isquemia miocárdica $(1,2)$.

La angina se define como el dolor, opresión o malestar, por lo general torácico, atribuible a la isquemia miocárdica transitoria. Es un concepto exclusivamente clínico y su diagnóstico se basa en las características y circunstancias que acompañan el dolor.

Las características que definen el dolor coronario son: el tipo, la localización, la irradiación, la duración, los factores desencadenantes y las circunstancias que lo alivian. En su forma habitual, los pacientes describen el dolor anginoso como una opresión, un peso o una sensación urente, localizado en la región retroesternal que irradia hacia los brazos, el cuello o la mandíbula.

Sin embargo, puede haber excepciones a esta descripción; en ocasiones se trata de una molestia indefinida que dificulta la respiración o bien se localiza exclusivamente en el cuello, la mandíbula, los brazos o incluso en las muñecas.

Los episodios de angina suelen iniciarse de forma gradual, alcanzan pronto su máxima intensidad y desaparecen también de forma paulatina en 1-10 min. En la angina de esfuerzo clásica, los síntomas presentan una clara relación con el ejercicio, en especial si éste se realiza tras las comidas o en un ambiente frío; las emociones pueden asimismo desencadenar la crisis. El cese de la actividad que provocó el dolor, el reposo o la administración de nitroglicerina sublingual determinan rápidamente su desaparición, excepto en algunas formas de angina (prolongada) en las que puede durar más de $30 \mathrm{~min}$. Un dolor de menos de $30 \mathrm{seg}$. de duración o continuo durante todo el día rara vez es de origen coronario (1).

\section{CASO CLÍNICO}

Paciente de 76 años de sexo femenino, que consulta en un Servicio de Urgencias hospitalario, por presentar dolor en área mandibular de tres días de evolución (Fig.1). Tras la exploración médica, la paciente fue remitida a su domicilio con el diagnóstico de 


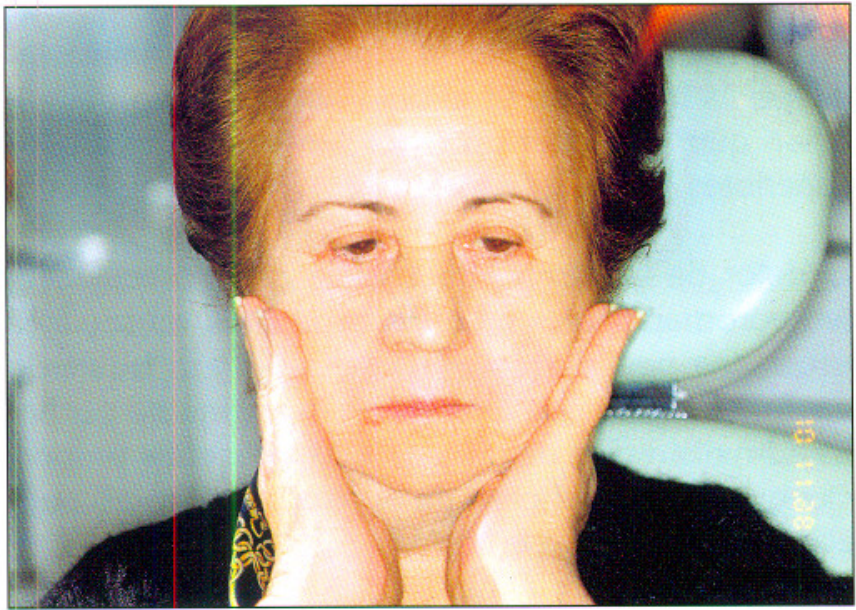

Fig. 1. Auto-imposición de manos para localizar el dolor.

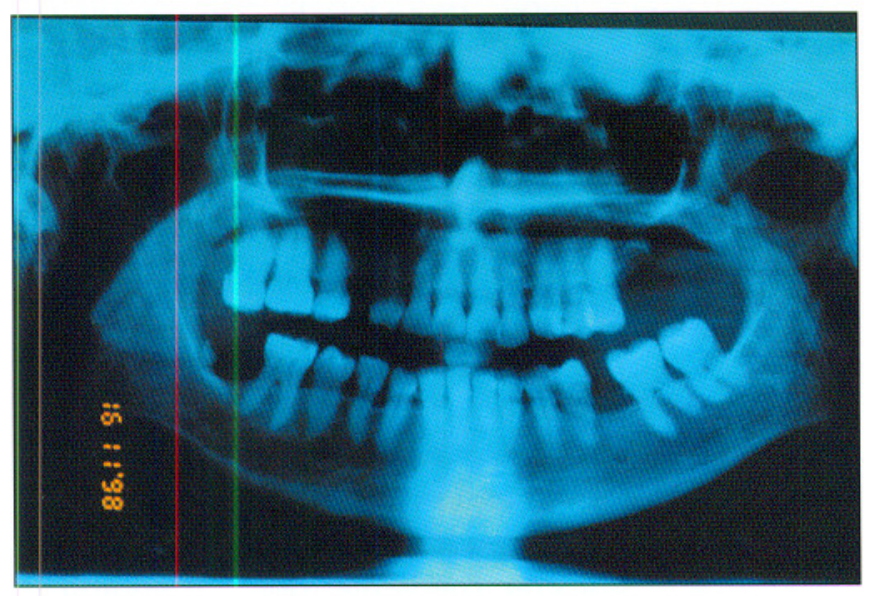

Fig. 2. Ortopantomografía de la paciente sin alteraciones significativas.

"artrosis mandibular" prescribiéndole analgésicos y recomendándole que acudiera al dentista.

Horas después la paciente acudió a nuestra consulta, donde le aplicamos el protocolo de estudio a pacientes con desórdenes temporomandibulares (DTM) y como examen complementario le practicamos una ortopantomografía (Fig. 2). Dentro de los antecedentes médicos personales de la paciente, cabe destacar que padecía hipertensión arterial desde hace 3 años y hipercolesterolemia desde hace un año.

En la anamnesis acerca de las características temporoespaciales del dolor, la paciente refiere que presenta episodios de dolor en región submandibular bilateral, de tres días de evolución, que ahora se irradia a zona precordial y a la garganta. Los episodios

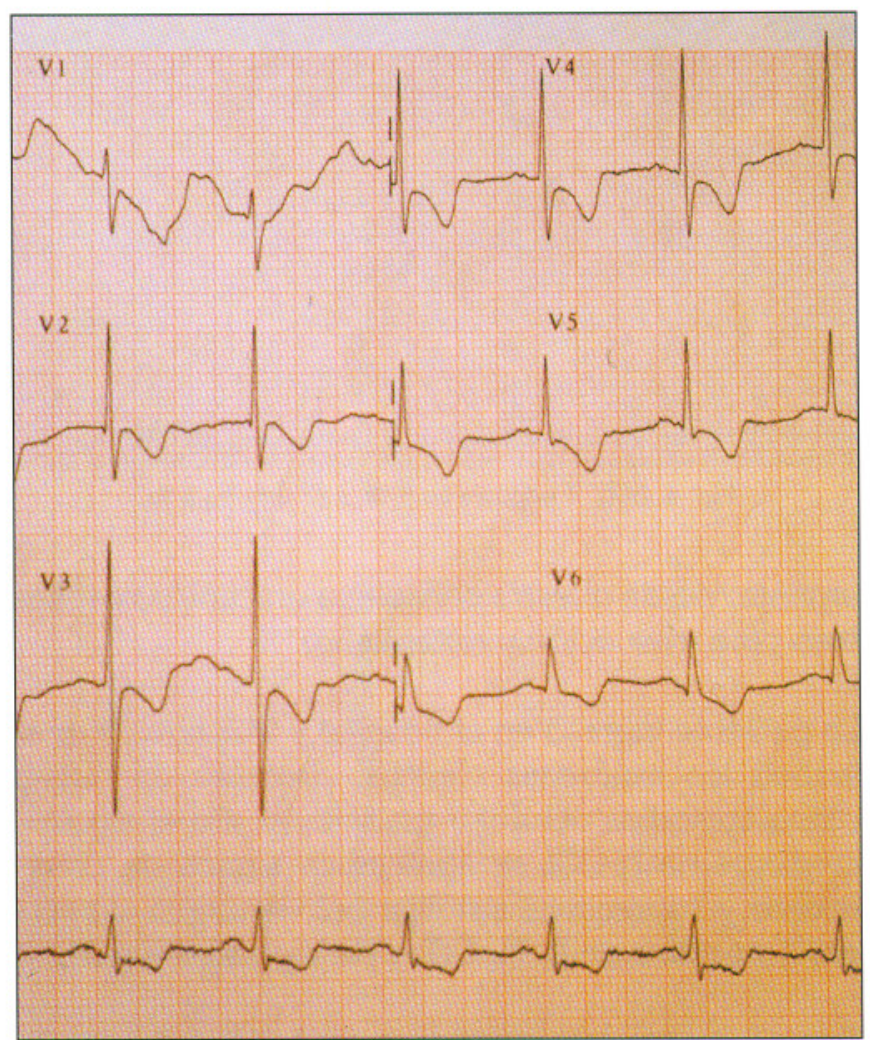

Fig. 3. ECG realizado cuando la paciente presentaba dolor mandibular como único síntoma.

de dolor tienen una duración que oscila entre los cinco y diez minutos, con sensación de falta el aire y sudoración acompañante. En este día ha presentado tres episodios. Este dolor ha sido resistente a los analgésicos que le prescribieron en el servicio de urgencias.

Tras la realización del examen estomatológico completo, encontramos un "Clic" de apertura en articulación temporomandibular derecha y crepitación en el lado izquierdo. Oclusalmente presentaba mordida cruzada y desviación de la línea media dental. La función mandibular estaba conservada con una apertura máxima de $39 \mathrm{~mm}$.

En la ortopantomografía se evidenciaban signos de artrosis leve en la articulación temporomandibular izquierda.

Estos hallazgos no justificaban la clínica del dolor que presenta la paciente, por lo que emitimos el diagnóstico clínico de presunción de "cardiopatía isquémica” y remitimos a la paciente, con carácter 


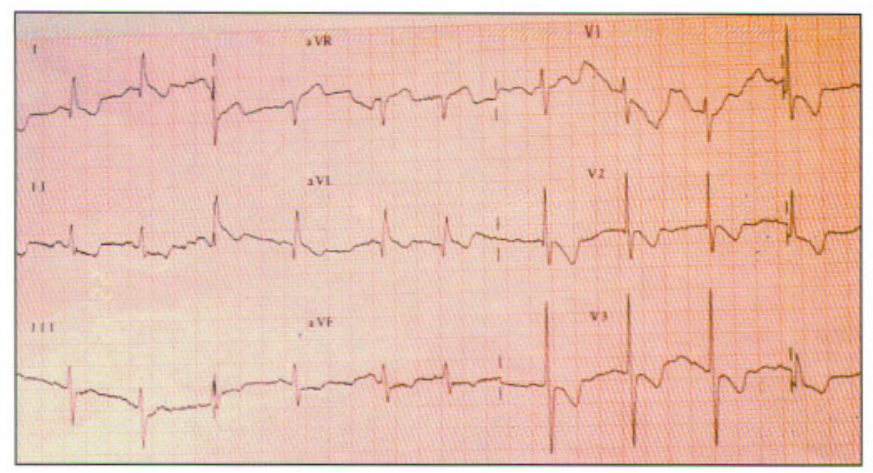

Fig. 4. ECG. T negativa en II, III, a VF y de $V_{4}$ a $V_{6}$.

urgente, desde nuestra consulta a un centro hospitalario para evaluación y tratamiento.

La paciente ingresó en el hospital y mientras le realizaban los exámenes clínicos, presento de nuevo varios episodios del dolor mandibular. En el electrocardiograma (ECG) se apreciaban signos de crecimiento y sobrecarga sistólica del ventrículo izquierdo, $T$ negativa en II, III, aVF y de $\mathrm{V}_{4}$ a $\mathrm{V}_{6}$ ( Fig 3).

Tras efectuarle los estudios complementarios pertinentes: análisis de sangre, radiografía de tórax, ecocardiograma y cateterismo diagnóstico, se encontró lesión severa en dos vasos coronarios.

Se le practicó angioplastia y a las tres semanas fue dada de alta con el diagnóstico de angor inestable (Grado III, B1), lesión severa de dos vasos coronarios y doble lesión aórtica degenerativa.

\section{DISCUSIÓN}

El dolor mandibular, como sitio de referencia de enfermedad cardiovascular o bien como como único síntoma inicial, en pacientes con isquemia cardiaca, ha sido referido por varios autores $(2,3,4)$.

El dolor orofacial de origen cardíaco se puede explicar por la existencia de fibras aferentes viscerales cardíacas que se unen con las fibras sensitivas somáticas de la región torácica superior y cervical, produciendo un dolor que comúnmente irradia al brazo $(5,6)$. La irradiación facial hacia el territorio de inervación trigeminal se observa con menor frecuencia y sería consecuencia de las interconexiones interneuronales entre las raíces cervicales superiores y los ni- veles medulares del nervio trigémino. La manifestación más frecuente, es el dolor en el ángulo mandibular que está inervado por las raíces cervicales superiores $\mathrm{C}_{2}$ y $\mathrm{C}_{3}(7,8)$.

El dolor de la artritis traumática de la articulación temporomandibular y de los espasmos musculares asociados puede irradiar a cuello y brazo izquierdo confundiéndose con el dolor de la angina de pecho. La administración de oxigeno y nitroglicerina proporcionará alivio en el caso de angina, pero no así para el dolor muscular o articular.

Otro factor a tener en cuenta en la presentación de un dolor cardiaco atípico, es la edad avanzada. El anciano parece haber reducido la percepción del dolor y muchas veces la isquemia cardiaca es silenciosa o cursa con sintomatología más inespecífica (9), nuestra paciente tenía edad avanzada además de antecedentes de hipertensión y arterioesclerosis.

\section{CONCLUSIONES}

Es fundamental realizar un buen diagnóstico diferencial del dolor orofacial, procurando encontrar el origen del mismo y teniendo muy presente que la cardiopatía isquémica se puede presentar inicialmente $o$ como único síntoma mediante dolor mandibular, dental, o como una cefalea (10).

La cualidad del dolor, duración, intensidad, localización, así como los factores desencadenantes o atenuantes nos facilitarán un correcto diagnóstico.

Los antecedentes médicos, así como la edad y el sexo del paciente son otro aspectos a tener en cuenta. Ante la sospecha de dolor de origen cardiaco el paciente debe ser remitido a un hospital urgentemente.

\section{BIBLIOGRAFÍA}

1. Sanz Romero G.A. Cardiopatía isquémica. En: Farreras y Rozman Medicina Interna. Décimo tercera edición en CD- Rom. Barcelona: Mosby Doyma SA; 1996. p. 533-42.

2. Kreiner, M, Okeson J. Toothache of cardiac ori- 
gin. Journal of orofacial pain. 1999; 13 (3): 201207.

3. Okeson JP. Dolor orofacial, según bell. $5^{\text {a }}$ edición. Barcelona: Quintessence; 1999.

4. Batchelder BJ, Krutchkoff DJ, Amara J. Mandibular pain as the initial and sole clinical manifestation of coronary insufficiency: Report of case. JADA 1987; 115: 710-712.

5. Peñarrocha Diago M. Dolor orofacial. Etiología, diagnóstico y tratamiento. Barcelona: Masson. S.A. 1997.

6. Tzukert A, Hasin Y, Sharav J. Orofacial pain of cardiac origin. Oral Surg 1981; 51: 484-486.

7. Graham LL, Schinbeckler GA. Orofacial pain of cardiac origin. JADA 1982; 104: 47-48.

8. Peñarrocha $M$, Silvestre FJ, Rodriguez $R$. Douleur faciale d'origine cardiaque. Rey Stomatol Chir maxillofac 1990; 91: 477-9.

9. Gregoratos G. Clinical manifestations of acute myocardial infarction in older patients. Am J Geriatr Cardiol 2001; 10(6): 345-7.

10. Auer J, Berent R, Lassnig E, Eber B. Headache as a manifestation of fatal myocardial infarction. Neurol Sci. 2001; Oct; 22(5): 395-7.

\section{CORRESPONDENCIA}

$M^{a}$ del Rosario Sáez Yuguero

Clínica Odontológica Universitaria

Hospital Morales Meseguer. Avda. Marqués de los Vélez, s/n.

30008 Murcia

Tf. 968 230061. Fax: 968239565

e-mail:mrosario@um.es 
